International Journal of Applied Mathematical Research, 1 (3) (2012) 282-287

(C)Science Publishing Corporation

www.sciencepubco.com/index.php/IJAMR

\title{
A New Approach To Improving The Estimate of Delta $(\Delta)$ Under European Option
}

\author{
Farshid Mehrdoust \\ Department of Applied Mathematics, Faculty of Mathematical Sciences,University of Guilan, Rasht, Iran \\ Email: fmehrdoust@guilan.ac.ir
}

\begin{abstract}
Estimating option sensitivities is another quite important task in financial mathematics. In this paper, we improve the estimate of $\Delta$ value for a vanilla European option by a robust stochastic algorithm based on quasi Monte Carlo methods and the antithetic variance reduction technique. In comparison to existing the naive Monte Carlo methods, we can improve accurate significantly by implementing our proposed algorithm.
\end{abstract}

Keywords: The Greeks, European Option, Quasi Monte Carlo, variance reduction

\section{Introduction}

One of the important things that a quant must do besides valuing options and knowing how to hedge them is to calculate the sensitivities of the values with respect to inputs. The main question is to judge and hedge the risks that one has incurred by entering the option. Note that we can compute the derivative of the price of a portfolio with respect to any of underlying parameters, and as with the match the derivative. The derivatives with respect to the various quantities are denoted by Greek letters. The derivatives are collectivity known as the Greeks. The Delta $(\Delta)$ is the most fundamental Greek which is increased from zero to one as a function of spot. In fact, at expiry time, a call option has payoff $(S-K)^{+}$. For $S<K$ the payoff has Delta equal to zero and for $S>K$, it has delta equal to one $[1,2,4]$. If $V\left(0, S_{0}\right)$ denotes the fair price at time 0 of a European call option with strike price $K$, the risk-neutral interest rate $r$, the 
stock's volatility $\sigma$ and expiry date $T$, then the Black-Scholes option valuation formula is:

$$
\begin{array}{r}
V\left(0, S_{0}\right)=S_{0} \Phi\left(\frac{\log \left(S_{0} / E\right)+\left(r+\frac{\sigma^{2}}{2}\right) T}{\sigma \sqrt{T}}\right)-E e^{-r T} \Phi\left(\frac{\log \left(S_{0} / E\right)+\left(r-\frac{\sigma^{2}}{2}\right) T}{\sigma \sqrt{T}}\right) \\
=S_{0} \Phi\left(d_{1}\right)-E e^{-r T} \Phi\left(d_{2}\right)
\end{array}
$$

where

$$
d_{1}=\frac{\log \left(S_{0} / E\right)+\left(r+\frac{\sigma^{2}}{2}\right) T}{\sigma \sqrt{T}}, \quad d_{2}=\frac{\log \left(S_{0} / E\right)+\left(r-\frac{\sigma^{2}}{2}\right) T}{\sigma \sqrt{T}}=d_{1}-\sigma \sqrt{T} .
$$

The standard option price sensitivities are the partial derivatives of $V=$ $V\left(0, S_{0}\right)$ with respect to these variables. Some of these partial derivatives are given special names and referred to:

$$
\begin{gathered}
\Delta=\frac{\partial V}{\partial S_{0}}(\text { delta }), \quad \Gamma=\frac{\partial^{2} V}{\partial^{2} S_{0}}(\text { gamma }) \\
\Theta=\frac{\partial V}{\partial T}(\text { thet }), \quad \rho=\frac{\partial V}{\partial r}(\text { rho }) \\
\left.v=\frac{\partial V}{\partial \sigma} \text { (vega }\right) .
\end{gathered}
$$

We note that $\Delta$ and $\Gamma$ measures the impact of price changes, $\Theta$ that of the decreasing time to maturity. $v$ and $\rho$ are measures for consequence of possible errors in the input parameters volatility and interest rate. In local or stochastic volatility models or even more advanced models, there is only the chance for obtaining the Greeks numerically. The most popular Greeks are course those are that are derived from Black-Scholes formula. In this paper, we will only focus on the computation of $\Delta$ for European call option and we propose a robust stochastic (RS) algorithm for estimating of $\Delta$.

\section{The Computation of $\Delta$}

We have a function $V\left(0, S_{0}\right)$, which in our case is the price of an option depending on the initial underlying asset price $S_{0}$, and the sensitivity we want to estimate is:

$$
\Delta=\frac{\partial V}{\partial S_{0}}=\lim _{\delta S_{0} \rightarrow 0} \frac{V\left(S_{0}+\delta S_{0}\right)-V\left(S_{0}\right)}{\delta S_{0}} .
$$

Since we are estimating the option price by quasi Monte Carlo simulation, the first approach coming to mind is to take sample paths and estimate $\Delta$ by the sample mean of finite differences between discounted payoffs. However, this approach is too naive and we will propose an efficient algorithm in the next section. 


\section{A Robust Stochastic Algorithm}

The slow probabilistic convergence of Monte Carlo estimators motivates for spending more effort in variance reduction techniques. Antithetic variates, control variates and importance sampling are approaches for increasing efficiency. Another way to accelerate convergence is to work with quasi random numbers (low discrepancy) rather than with pseudo random numbers. The key of robust stochastic (RS) algorithm is to use of Halton sequence [3] and Box-Muller method $[5,6]$ in antithetic variance reduction technique. Halton sequences use the representation of integers in another base to produce numbers between 0 and 1. For each dimension another base is used.

\section{Theorem:}

Consider the stochastic ordinary differential equation, $d S_{t}=r S_{t} d t+\sigma_{t} S_{t} d W_{t}$ with a change of sign in the term containing the Brownian motion. Then the following stochastic process, $\Upsilon_{t}$ yields a variance reduction.

$$
\Upsilon_{t}=\frac{e^{-r t}}{2}\left[\left(S_{t}-K\right)_{+}+\left(S_{t}^{-}-K\right)_{+}\right]
$$

Proof. We have

$$
\begin{gathered}
d S_{t}=r S_{t} d t+\sigma_{t} S_{t} d W_{t} \\
d S_{t}^{-}=r S_{t}^{-} d t-\sigma_{t} S_{t}^{-} d W_{t}
\end{gathered}
$$

Then

$$
E\left[\Upsilon_{t}\right]=E\left[e^{-r t}\left(S_{t}-K\right)_{+}\right], \quad C_{0}=E\left[\Upsilon_{T}\right]
$$

Because $S_{t}$ and $S_{t}^{-}$have the same law, we can write

$$
\begin{gathered}
\operatorname{var}\left[\Upsilon_{t}\right]=\frac{e^{-2 r t}}{4}\left\{\operatorname{var}\left[\left(S_{t}-K\right)_{+}\right]+\operatorname{var}\left[\left(S_{t}^{-}-K\right)_{+}\right]\right\}+ \\
+\frac{e^{-2 r t}}{2} E\left[\left(\left(S_{t}-k\right)_{+}-E\left(S_{t}-K\right)_{+}\right)\left(\left(S_{t}^{-}-K\right)_{+}-E\left(S_{t}^{-}-K\right)\right)\right] \leq \frac{1}{2} \operatorname{var}\left[e^{-r t}\left(S_{t}-K\right)_{+}\right] .
\end{gathered}
$$

Since the function $f(x)=(S-K)_{+}$is monotone and two variables $S_{t}$ and $S_{t}^{-}$ are negatively correlated. Therefore, we have obtained a new process with the same expectation and with a smaller variance.

Now, based on the above theorem, we employ the Halton sequences and BoxMuller method for construction the following algorithm.

\section{The RS algorithm}

(1) H1 = GetHalton(ceil(NPoints/2),Base 1) 
(2) H2 = GetHalton(ceil(NPoints/2),Base 2)

(3) $\operatorname{VLog}=\operatorname{sqrt}\left(-2^{*} \log (\mathrm{H} 1)\right)$

(4) Norml $=\operatorname{VLog} * \cos \left(2^{*} \mathrm{pi}^{*} \mathrm{H} 2\right)$;

(5) Norm $2=\operatorname{VLog} * \sin \left(2 * \mathrm{pi}^{*} \mathrm{H} 2\right)$

(6) Norm $=[$ Norm l; Norm 2]

(7) Payoff $\mathrm{l}=\max \left(0, \mathrm{~S} 0 * \exp \left(\mathrm{nuT}+\mathrm{siT}^{*}\right.\right.$ Norm $\left.)-\mathrm{K}\right)$

(8) Payoff $2=\max \left(0,(\mathrm{~S} 0+\mathrm{dS}) * \exp \left(\mathrm{nuT}+\mathrm{siT}^{*}\right.\right.$ Norm $\left.)-\mathrm{K}\right)$

(9) Payoff $3=\max \left(0, \mathrm{~S} 0 * \exp \left(\mathrm{nuT}+\mathrm{siT}^{*}{ }_{-N}\right.\right.$ Norm $\left.)-\mathrm{K}\right)$

(10) Pay $1=($ Payoff $1+$ Payoff 3$) / 2$

(11) Payoff $4=\max \left(0,(\mathrm{~S} 0+\mathrm{dS}) * \exp \left(\mathrm{nuT}+\mathrm{siT}^{*}\right.\right.$-Norm $\left.)-\mathrm{K}\right)$

(12) Pay $2=($ Payoff $2+$ Payoff 4$) / 2$

(13) $\mathrm{AVMCP}=\exp \left(-\mathrm{r}^{*} \mathrm{~T}\right) *($ Pay1-Pay2)

(14) SampleDiff $=\exp \left(-r^{*} T\right) *($ Pay2-Pay1 $) / d S$

\section{Numerical Experiments}

In order to illustrate the performance of our proposed algorithm, we compute $\Delta$ under European call option with $S_{0}=80, K=100, r=0.1, T=1, \sigma=0.3$ and $d S=0.001$. Table 1 and Figures 1 and 2 show test results of a comprehensive study on European call option. As we can see in Figure 1 and 2, we are able to obtain a significant improvement by using RS algorithm in comparison to naive stochastic method (NS). 


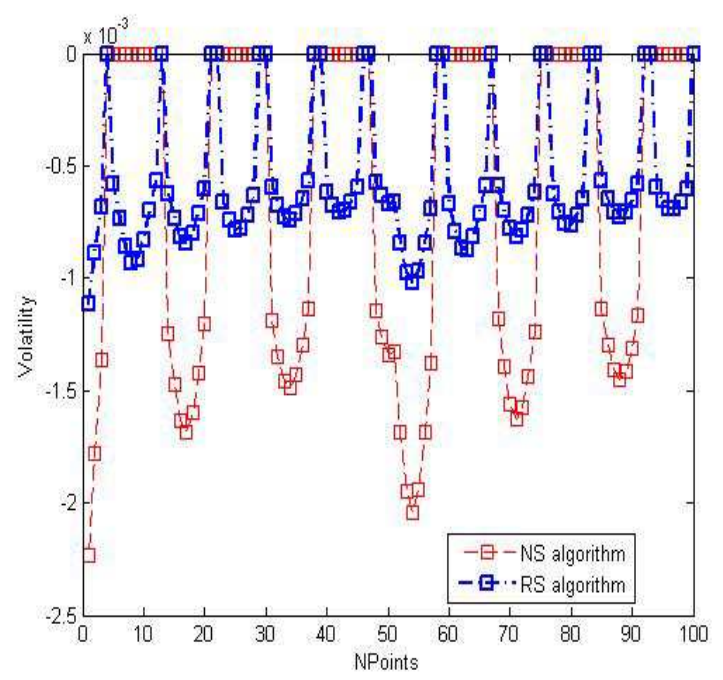

Figure 1: Comparison of standard deviation between RS and NS algorithms

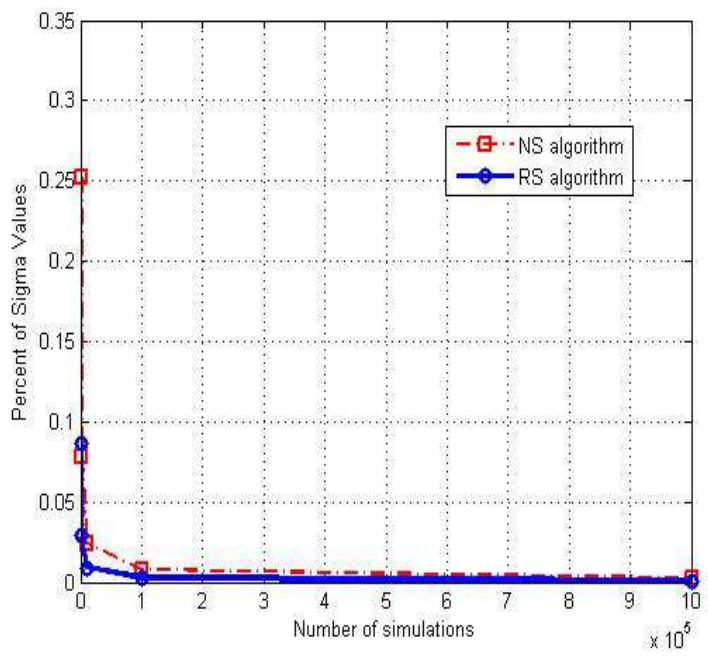

Figure 2: Comparison of the volatility between RS and NS algorithms

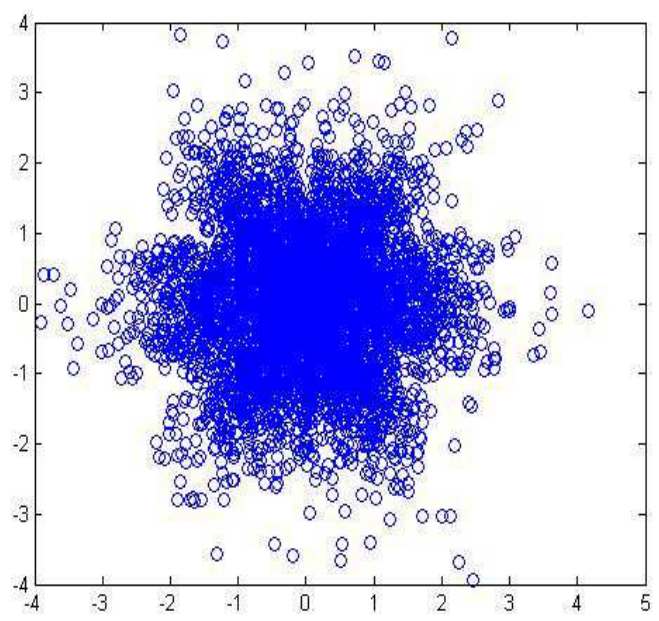

Figure 3: Simulation of normal random numbers with Box-Muller method 
Table 1: The values of $\Delta$ and $\sigma$ with the RS and NS algorithms

\begin{tabular}{|c|c|c|c|c|}
\hline Number of Replications & NS- $\Delta$ & NS- $\sigma$ & RS- $\Delta$ & RS- $\sigma$ \\
\hline 100 & 0.5495 & 0.2521 & 0.5597 & 0.0862 \\
\hline 1000 & 0.5730 & 0.0778 & 0.5344 & 0.0288 \\
\hline 10000 & 0.5314 & 0.0245 & 0.5312 & 0.0092 \\
\hline 100000 & 0.5309 & 0.0077 & 0.5308 & 0.0029 \\
\hline 1000000 & 0.5308 & 0.0024 & 0.5308 & $0.1448 \times 10^{-4}$ \\
\hline
\end{tabular}

\section{Conclusion}

A new algorithm for improving the estimate of $\Delta$ was derived and tested. The method was based on antithetic variance reduction procedure, Halton points and the Box-Muller methods.

\section{References}

[1] P. Brandimarte, Numerical Methods in Finance and Economics, John Wiley \& Sons, 2006.

[2] D. J. Duffy, Finite Difference Methods in Financial Engineering, John Wiley \& Sons, 2006.

[3] C. Lemieux, Monte Carlo and Quasi-Monte Carlo Sampling, Springer, 2009 .

[4] G. Levy, Computational Finance, Elsevier, 2004.

[5] H. Osnes, Variance reduction techniques for Monte Carlo simulation, SINTEF and University of Oslo, 1997.

[6] R.Y. Rubinstein, Simulation and the Monte Carlo Method, John Wiley \& Sons, 1981. 